\title{
Mechanical Bridge to Decision: What Are the Options for the Management of Acute Refractory Cardiogenic Shock?
}

\author{
Daniel Goldstein • Siyamek Neragi-Miandoab
}

Published online: 16 December 2010

(C) Springer Science+Business Media, LLC 2010

\begin{abstract}
While great strides have been made in the management of heart failure syndromes, acute refractory cardiogenic shock carries a dismal prognosis. Initial treatment with inotropes and balloon counterpulsation can restore hemodynamics, but many patients deteriorate and succumb to multisystem organ failure if timely mechanical circulatory support is not established. Institution of support is intended as a life-saving measure where the final treatment strategy remains uncertain. This scenario is referred to as "bridge to decision." Notably, most of these patients present to community hospitals, where advanced mechanical support technologies are scarce or nonexistent. Delays in referral to a tertiary center contribute to the bleak outcomes. Herein, we review the initial management of acute heart failure and refractory cardiogenic shock, profile the typical patient, delineate current options for mechanical support in patients with acute refractory cardiogenic shock, and propose suggestions for the establishment of a seamless transfer process of these ill patients to tertiary centers.
\end{abstract}

Keywords Mechanical support - Ventricular assist device . Acute heart failure $\cdot$ Bridge to decision . Bridge to recovery $\cdot$ Bridge to transplantation $\cdot$ Refractory cardiogenic shock

\section{Introduction}

The syndrome of acute heart failure is well recognized and is defined as the rapid onset of signs and symptoms

D. Goldstein $(\bowtie) \cdot$ S. Neragi-Miandoab

Department of Cardiovascular and Thoracic Surgery, Montefiore

Medical Center, Albert Einstein College of Medicine,

3400 Bainbridge Avenue, MAP 5,

Bronx, NY 10467, USA

e-mail: dgoldste@montefiore.org secondary to abnormal cardiac function. Acute heart failure may be related to systolic or diastolic dysfunction, abnormal cardiac rhythms, or preload and/or afterload mismatch [1]. It may involve either or both ventricles and may be precipitated by cardiac or noncardiac factors (eg, thyrotoxicosis, lack of medical compliance, or septicemia). In most instances, it is life-threatening and requires urgent treatment. Common causes of acute heart failure include acute coronary syndromes, decompensation of preexisting chronic heart failure, acute arrhythmias, acute valvular regurgitation, and postcardiotomy shock.

Initial management of acute heart failure is best provided by a multidisciplinary team expert in the management of these patients and includes appropriate monitoring of vital signs, ventilatory assistance as needed, and medical treatment that may incorporate morphine, anticoagulants, vasodilators, and diuretics. In patients whose hemodynamic condition deteriorates (ie, peripheral vasoconstriction, hypotension, and end-organ dysfunction), inotropic therapy to augment forward cardiac output, typically $\beta$-agonists and/ or phosphodiesterase inhibitors, is instituted. When the combination of inotropic support and fluid management fails to restore blood pressure and end-organ perfusion, vasopressor therapy is introduced. This usually includes addition of $\alpha$-agonists and/or vasopressin and can be accompanied by intra-aortic balloon pump (IABP) counterpulsation. For those situations in which effective established interventions exist (eg, percutaneous angioplasty for myocardial infarction, surgical correction of postinfarct ventricular septal defect), prompt referral for definitive therapy is undertaken. Discussion of these entities is beyond the scope of this chapter.

For patients with acute heart failure who are unresponsive to the conventional medical therapies described above (ie, those with refractory cardiogenic shock) and for whom no other definitive therapies exist, institution of mechanical 
circulatory support remains the only option for survival. Precise estimates of incidence and prevalence of this entity are scarce but the ADHERE (Acute Decompensated Heart Failure National Registry [2]) multicenter database suggests that of acute heart failure admissions in the United States, $2 \%(100,000)$ of patients annually are high risk $(20 \%$ inhospital mortality) and likely represent cardiogenic shock patients.

While the need to institute mechanical support often is clear, the final therapeutic strategy often is uncertain. Institution of advanced mechanical support (ie, beyond IABP) under these circumstances is termed "bridge to decision" and it underscores the vagueness that often clouds ultimate management of these patients. Application of circulatory support is intended to rapidly stabilize hemodynamics and restore end-organ perfusion. Achievement of these critical goals provides time to assess the potential for myocardial recovery and device removal, to evaluate neurological status (which may have been compromised by hypoperfusion), and to establish candidacy for bridging to long-term implantable ventricular assist device (VAD) support or, rarely, directly to transplantation. Not uncommonly, underlying conditions are uncovered (eg, patient's documented prior wishes, malignancy) or complications develop (eg, massive stroke, hypoxic encephalopathy, irreversible renal failure) that preclude further therapeutic measures and ultimately culminate in the patient's demise from disease progression, complications of mechanical therapy, or withdrawal of support.

For those fortunate patients in whom circulatory stability can be maintained, efforts firstly are focused on assessing potential for ventricular recovery and device explantation. While evidence of myocardial recovery is sought, parallel evaluation of neurological status, degree of end-organ dysfunction, and identification of social supports are undertaken. Should sufficient myocardial recovery be documented, weaning and explantation of mechanical support ensues. Optimal medical therapy and close follow-up of these patients is mandatory. For patients in whom myocardial recovery does not occur and no other contraindications exist, long-term support with an implantable VAD is seriously considered as prolonged bridge to recovery, bridge to transplantation, or destination therapy.

Perhaps the greatest hindrance to wider application of temporary bridge-to-decision mechanical support, and hence, to better outcomes, lies in the fact that most of these patients first present in community hospitals that lack the expertise and resources to institute these potentially lifesaving therapies.

Rural hospitals or those located in smaller metropolitan communities can only provide ventilatory assistance and perhaps basic inotropic/pressor support. Medium-sized community hospitals with or without catheterization laboratories may in addition provide IABP support and more sophisticated knowledge in the use of inotropic/pressor agents, while tertiary care institutions with established cardiac surgical programs can provide circulatory support with extracorporeal membrane oxygenation (ECMO) and/or temporary extracorporeal support devices. However, even in the latter category of hospitals, limited surgical experience with institution and management of mechanical support often leads to delays in optimal treatment and referral of refractory shock patients, with predictable poor outcomes. Therefore, it is critical for all three categories of "spoke" hospitals to establish a referral relationship with "hub" institutions with transplantation and mechanical assistance programs that can facilitate seamless transfer and prompt establishment of mechanical support for these critically ill patients [3-5].

It is important to emphasize that while the role of implantable systems in the management of highly selected heart failure subgroups is a guideline-recommended option [6], the use of temporary mechanical assistance, generally thought to be the only option for survival for patients in refractory cardiogenic shock, has not been submitted to randomized clinical evaluation, and its level of efficacy remains a Class IIb, level of evidence B in the European Society of Cardiology guidelines [1]. The use of long-term implantable systems, while associated with excellent hemodynamic support, generally is not considered in these acutely ill patients because of the uncertainty regarding neurologic injury and irreversible end-organ damage, either of which precludes implantation of these expensive and complex support systems.

\section{Bridge to Decision: Who, What and When?}

The goal of bridge-to-decision mechanical support is to provide rapid and full circulatory support for patients in decompensated cardiogenic shock who have at least reasonable potential for meaningful survival. In certain situations, the decision of whether to offer this advanced therapy can be difficult. A young patient who has suffered a substantial period of hypoperfusion (eg, $45 \mathrm{~min}$ of cardiopulmonary resuscitation with chest compressions) in whom mechanical support may restore hemodynamics and perhaps end-organ function, but whose neurological prognosis is dim, is one such scenario. The patient for whom no long-term option (destination therapy or transplantation) exists but whose family wants "everything to be done" because they are not ready to face a loved one's untimely death, represents a difficult challenge. In these situations, involvement of multidisciplinary teams, including palliative 
care and ethics specialists as well as clergy, is of utmost importance.

Once the decision has been made to offer temporary mechanical support, several factors will determine the system, access site, and configuration for deployment. Patient-related factors include the presence of a healed median sternotomy, extent of biventricular dysfunction, quality of femoral and right axillary arteries, previous inferior vena cava filter, presence of coagulopathy, and endorgan dysfunction. Surgeon-related factors include familiarity with available systems and desirability to take on the challenge. Other factors include operating room, catheterization lab, and intensive care unit availability, in addition to the trained staff needed to implant and care for the mechanically supported patient.

In general, the available device(s) will dictate the approach to undertake. Broadly speaking, the longer the period of shock, the greater the extent of end-organ dysfunction, and postcardiotomy shock etiology all strongly favor institution of biventricular support. This strategy provides maximal control of the circulation with optimal end-organ perfusion. It often averts the need of high-dose pressors that, while maintaining perfusion pressure, do so at the expense of peripheral and visceral vasoconstriction. An added benefit of biventricular support is that an oxygenator is readily connected to the right ventricular assist device (RVAD) circuit should respiratory failure ensue.

Early presentation, hemodynamics suggestive of preserved right ventricular function (low central venous pressure, high right ventricular stroke work index [7, 8] and favorable echocardiographic indices of right heart function [9]), and normal end-organ function all suggest the possibility of stabilization with univentricular (left ventricular assist device [LVAD] only) support. Nonetheless, the inability to accurately predict right ventricular failure after LVAD support mandates that the surgeon be prepared to insert a RVAD at the index operation. Delay in instituting RVAD support has been associated with poor outcomes [10•].

Type of access for institution of mechanical support can be peripheral (via femoral artery/femoral vein or axillary artery/femoral vein) or central (via median sternotomy with direct cannulation of the appropriate cardiac chambers and greater vessels). Certain scenarios suggest an advantage of one approach over another. The refractory shock patient with hepatic failure and severe coagulopathy is at very high surgical risk and, hence, may be best served by institution of peripheral circulatory support. On the other hand, peripheral cannulation through a femoral artery is contraindicated with known peripheral vascular disease. A large patient (body surface area $>2 \mathrm{~m}^{2}$ ) may be best served by the higher VAD flows that can best be obtained by central cannulation.

\section{Currently Available Short-term Support Systems}

Surgically Implanted Systems

\section{Continuous-flow Pumps}

All extracorporeal, surgically implanted, continuous-flow pumps rely on centrifugal flow motion. Centrifugal pumps (Bio-Pump [Medtronic Inc, Brooklyn Park, MN] and Sarns [Terumo Cardiovascular Systems Corporation, Ann Arbor, $\mathrm{MI}$ ) are familiar assist systems because of their routine use in cardiopulmonary bypass. Designed for short-term support, they are used in several modalities including ECMO or bridging to transplant or a more sophisticated VAD (so called bridge to bridge). Although many different pumphead designs are available, the differences in design of the pump heads are in the numbers of impellers, the shape and angle of the blades, and the priming volume [11]. Most pumps consist of a single moving part so they are disposable and relatively inexpensive to manufacture. Centrifugal pumps work on the principle of imparting momentum to fluid by operation of moving blades, impellers, or concentric cones [11, 12]. These pumps generally provide high flow rates at low pressures. The amount of flow generated is sensitive to afterload and filling pressures.

Institution of mechanical support with these pumps can occur via connection to cannulae placed centrally or peripherally for ECMO support (femoral vein to femoral artery with intervening oxygenator). RVAD support can be established easily via cannulae placed through purse-string sutures into the right atrium and pulmonary artery. LVAD support is attained by cannulation of the left atrium or left ventricular apex and the ascending aorta. Often, this can be accomplished without the aid of cardiopulmonary bypass.

Typically, bioactive surfaces used in the tubing circuit allow delayed administration of intravenous heparin immediately after implant. This delay provides time for hemostatic mechanisms to return to baseline before institution of systemic anticoagulation. To avoid stasis and thrombus formation, activated clotting times (ACT) are maintained at 150 to $250 \mathrm{~s}$. In the event of extensive fibrin deposition or frank pump thrombosis, bedside exchange of these pumps can be readily achieved. Like all systems herein described, complications associated with this technology are related to the severity of the shock state and include bleeding, coagulopathy, pulmonary and renal insufficiency, and neurological deficits. Pump failure is very rare [13].

CentriMag (Levitronix, Waltham, MA) This particular device has been introduced over the past few years as a new generation of centrifugal pump. Unlike the aforementioned systems, the motor in this particular system levitates 
magnetically so that rotation can be achieved without friction, areas of stasis, or component wear during operation. This results in lower risk for hemolysis and thrombus formation within the pump [14] and reduced likelihood of pump replacement. Like its predecessors, this pump can be connected to centrally placed cannulae for univentricular or biventricular support, or to peripherally placed cannulae for ECMO support.

John et al. [14] described their experience with 12 patients who underwent institution of biventricular CentriMag support as a bridge to decision. Most patients were implanted in the setting of post-myocardial infarction cardiogenic shock, had impaired neurological responsiveness, were refractory to IABP support, and had evidence of multisystem organ dysfunction [14]. Of the 12 patients, 8 were successfully bridged to a long-term implantable device. Two patients recovered sufficient cardiac function to allow explantation and two patients expired shortly after implant. Haj-Yahia and colleagues [15] described four patients with end-stage cardiomyopathies who underwent CentriMag support as a bridge to decision. After recovery of end-organ function and a mean support time of 43 days without neurologic or thromboembolic complications, all four patients were successfully bridged to transplantation. Loforte et al. [16] reported on the use of this system in 30 patients with acute cardiogenic shock and biventricular failure; 15 were successfully bridged to a long-term device without cannula exchange. De Robertis and colleagues [17] described their experience with the CentriMag system as a bridge to decision in 16 young moribund patients with shock refractory to IABP support; $11(68.7 \%)$ were successfully bridged to recovery, long-term support, or transplantation with a 1-year survival of $65 \%$. No instances of device failure were reported. The system has been used as a bridge to decision to support pediatric patients as well [18].

In summary, extracorporeal centrifugal pump technology has a long history in the field of cardiac surgery and is very effective in restoring hemodynamics and end-organ perfusion for patients with acute refractory shock. The CentriMag system, in particular, allows delayed institution of systemic anticoagulation, and reduced heparinization appears to be well tolerated as long as pump flows can be consistently maintained above $4 \mathrm{~L} / \mathrm{min}$ [14]. Pump thrombosis and pump failure are extremely rare and prolonged periods of support without pump exchange are possible.

\section{Pulsatile-flow Systems}

Abiomed BVS 5000 and AB5000 (Abiomed Inc., Danvers, MA) The details of the BVS 5000 support system have been presented [19]. Briefly, the BVS 5000 is an extracorporeal pulsatile device driven by a pneumatic drive console and designed for use as a bridge to recovery. It is capable of providing univentricular or biventricular support. A transport console allows horizontal placement of the pump, facilitating transfer of these acutely ill patients to transplant centers. Limitations are similar to those inherent to most centrifugal pumps and include patient immobility, less than 2-week support times, need for anticoagulation, and the need for reoperation for device removal [12]. Moreover, about $600 \mathrm{~mL}$ of blood volume remain in the extracorporeal tubing at all times, thereby increasing the incidence of coagulopathy and rendering the system prone to stasis and fibrin deposition.

As a result of these limitations, the Abiomed AB5000 was introduced. It is the ambulatory version of the BVS blood pump [20]. Its paracorporeal position eliminates the large column of extracorporeal blood. The pump housing holds about $100 \mathrm{~mL}$ of blood and is clear, allowing for visualization of clot. It is intended for short-term to intermediate-term use. Recipients must have a BSA over $1.3 \mathrm{~m}^{2}$. The inflow and outflow cannulae must be placed transthoracically, but because of compatibility with the BVS 5000, the latter can be exchanged for the AB5000 at the bedside without another operation. Both systems are easy to wean at the bedside to allow for evaluation of ventricular recovery.

Like the centrifugal pumps, the Abiomed systems require systemic heparinization to maintain an $\mathrm{ACT}$ of 180 to $200 \mathrm{~s}$. The systems differ from centrifugal pumps in that the proprietary outflow cannulae (to the pulmonary artery [RVAD] and/or ascending aorta [LVAD]) require suture anastomosis of the outflow graft to the recipient vessel. Therefore, the system often requires institution of cardiopulmonary bypass and frequently is associated with bleeding-related complications. Anecdotally, the system has been used with peripheral access (femoral artery and vein) when central cannulation was not possible [21]. These systems have been used successfully to bridge patients to recovery, long-term implantable devices, or transplantation.

\section{Percutaneous Systems}

\section{TandemHeart (CardiacAssist Inc., Pittsburgh, PA)}

The TandemHeart system is a percutaneous extracorporeal cardiopulmonary support device. It consists of an external, centrifugal, continuous flow pump and two cannulae [22]. While the actual pump is not much different than those described previously, the ability to percutaneously deploy this device in a univentricular or biventricular configuration renders this technology unique. The inflow catheter is inserted via the femoral vein into the right atrium and then passed transseptally (under fluoroscopic and transesopha- 
geal guidance) into the left atrium and secured in place. The outflow is provided through a cannula placed in the femoral artery, thereby establishing left atrial-to-femoral artery bypass. Percutaneous RVAD support also can be achieved with cannulation of the right atrium and pulmonary artery via the right internal jugular and femoral veins (Fig. 1). In the percutaneous configuration, up to $4 \mathrm{~L}$ of blood flow per minute can be obtained. Similar to previously described support systems, the TandemHeart VAD can be placed via standard open central cannulation with resultant higher flows and is easy to wean at the bedside.

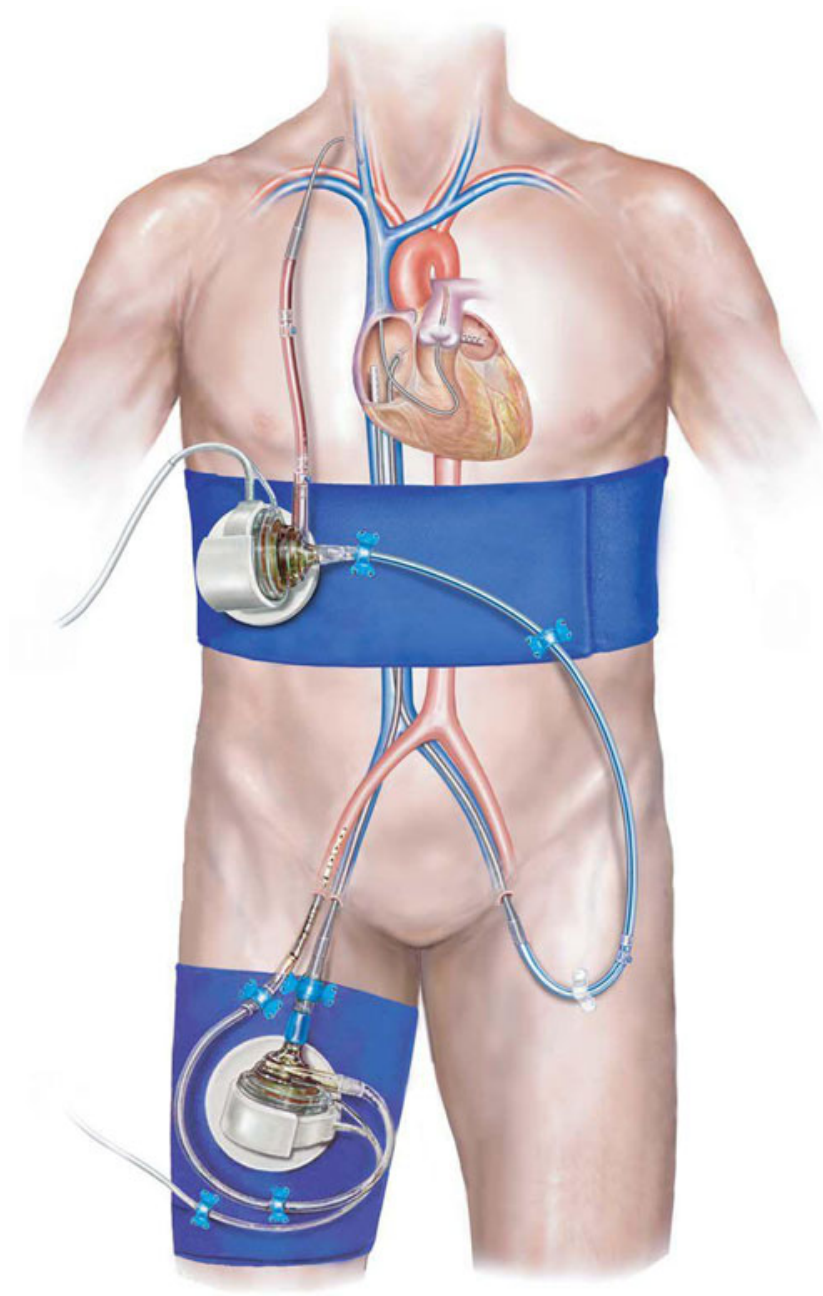

Fig. 1 Percutaneous biventricular assist device support with the TandemHeart (CardiacAssist Inc., Pittsburgh, PA). The device is deployed under fluoroscopic and transesophageal guidance. RVAD support is attained with an inflow cannulae placed into the right atrium via the femoral vein and an outflow cannula placed into the pulmonary artery via the right internal jugular vein. LVAD support is achieved with an inflow cannula advanced from the right femoral vein into the right atrium and across the interatrial septum into the left atrium. The outflow cannula is seen entering the right femoral artery. Typically, flows of 3.5 to $4 \mathrm{~L} / \mathrm{min}$ can be obtained (Image provided courtesy of CardiacAssist Inc., Pittsburgh, PA, with permission). LVAD left ventricular assist device; $R V A D$ right ventricular assist device
The most frequent use of the TandemHeart in the percutaneous configuration has been in the catheterization lab as prophylactic support for patients undergoing highrisk percutaneous interventions. It provides short-term support from a few hours up to 3 weeks, giving the heart time to declare recoverability [23]. The device can be extracted via the femoral vessels at the bedside. The residual atrial septal defect usually is of no consequence. If needed, the device cannulae can be converted and connected to a cardiopulmonary bypass machine [24]. The disadvantages include mandatory anticoagulation, occasional thrombocytopenia, and patient immobility, similar to all aforementioned systems. Furthermore, establishing percutaneous LVAD support requires a transseptal puncture, which requires special training.

The largest series reporting the results with this system in patients in acute refractory cardiogenic shock consisted of 117 patients with shock refractory to IABP support and vasopressor therapy, nearly half of whom had undergone active cardiopulmonary resuscitation [25*]. Average duration of support was nearly 6 days. Immediate improvement in blood pressure, mixed venous saturation and end-organ function was noted. Survival at 30 days was a remarkable $60 \%$. Brinkman et al. [26] described the percutaneous use of this system in 11 bridge-to-decision patients (indeterminate neurological status or multisystem organ failure). Length of support ranged from 1 to 11 days. Of 11 patients, 7 had support withdrawn, 2 were bridged to long-term LVADs, 1 was bridged directly to transplant, and 1 patient with an acute myocardial infarction was explanted for recovery. No device failures occurred. The authors concluded that the percutaneous TandemHeart VAD system allowed time for neurological and end-organ status evaluation without the added expense and morbidity of a long-term VAD [26].

Impella Recover System (Abiomed Inc., Danvers, MA)

One of the newest VADs on the market is the Impella Recover system. The device is a microaxial pump that is placed in retrograde fashion across the aortic valve into the left ventricle. Blood is aspirated from the left ventricular cavity and ejected into the ascending aorta, thereby establishing a left ventricular-to-aortic bypass. The axial flow pump contains a rotor that is driven by an electrical motor. The pump is continuously purged with a heparin solution that prevents pump thrombus and reduces the need for systemic heparinization. It can provide circulatory assistance for up to 5 days. A driveline connects to a small console that has 10 different speed settings (2000 to $50,000 \mathrm{rpm}$ ) and reads the pressure difference between the aortic blood and the left ventricle [27].

Implantation of the system requires femoral arterial access. The Impella LP 2.5 system is the only truly percutaneous 
system, deployed through a 13-French sheath (Fig. 2). It can deliver $2.5 \mathrm{~L} / \mathrm{min}$ of flow. The larger LP 5.0 system requires a surgical cutdown for placement (21 French); hence, it is not truly percutaneous. Two other versions of this miniaturized pump exist. The Impella LD system is placed through a graft sewn to the ascending aorta and, thus, usually is reserved for postcardiotomy shock patients. A fourth system, the Impella $\mathrm{RD}$, is intended for right ventricular support and is not available at this time in the United States.

Several advantages of these systems are worth emphasizing. They are relatively easy to deploy, do not require transseptal puncture, and use reduced systemic heparin. Weanability is easily assessed with the dynamic pump stop feature. Aortic valve injury and device-induced aortic regurgitation are extremely rare. Recently, spoke-to-hub transport for a patient with the LP 5.0 system was reported [28]. The LP 5.0 system was implanted in five patients with post-myocardial infarction cardiogenic shock via a right axillary approach, thereby avoiding median sternotomy and complications related to femoral artery cannulation [29].

Disadvantages include the inability to deploy in patients with severe peripheral vascular disease or mechanical aortic prostheses, inability to support the right ventricle, short duration of support, and the possibility of cannula dislodgement and limb ischemia.

A recent review from a single center comparing outcomes of patients in cardiogenic shock receiving ECMO

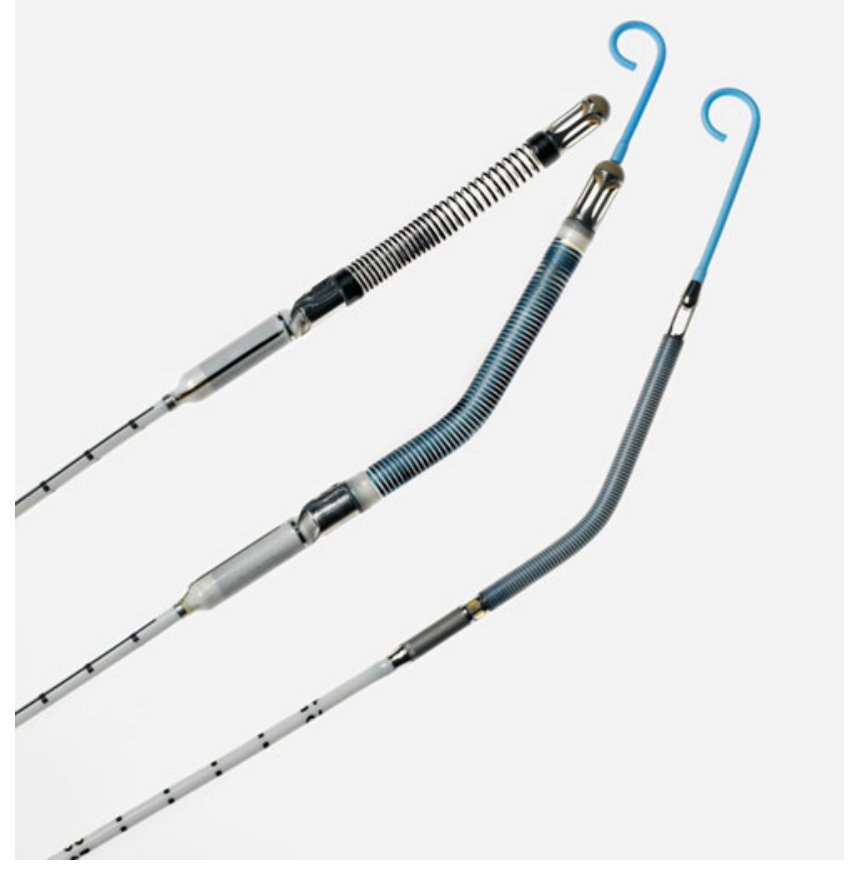

Fig. 2 Impella family of devices. From left to right: 5.0 left direct (no pigtail); 5.0 LP; and 2.5. (Image provided by ABIOMED, Inc., with permission) support or Impella LP 5.0 assistance demonstrated similar survival, although arterial thrombosis and blood transfusions were less frequent in Impella recipients [30]. A randomized clinical trial comparing Impella 2.5 to IABP for 26 patients with post-myocardial infarction cardiogenic shock demonstrated superior hemodynamic support for the Impella device but no difference in 30-day survival [31•].

\section{Creating an Optimal Spoke-Hub Environment}

A major impediment to better outcomes in acute refractory cardiogenic shock rests in the fact that most of these events occur in nontransplant medical centers where sophisticated mechanical support systems either do not exist or are seldom used. Therefore, it is essential that these medical centers establish a relationship with a transplant/VAD center. These spoke-and-hub relationships are largely based on geographical proximity.

It has been suggested that patients requiring mechanical support be transferred as soon as possible to a hub center, regardless of whether the spoke institution has an active cardiac surgical program [4]. Many skilled surgeons outside of a transplant/VAD referral center lack the experience in managing these complex patients, and are unlikely to have at their disposal a variety of systems to tailor the type of circulatory support to the individual patient. Moreover, cardiology expertise in advanced heart failure, access to clinical trials, and knowledge in weaning these devices is usually unavailable in spoke institutions. Therefore, we believe that patients with acute refractory shock should be transferred readily and seamlessly to a transplant/VAD hub center.

Several factors are of paramount importance in creating an optimal environment for the management of these patients. A team within the spoke institution that will exclusively manage these patients until they can be transferred to the hub institution must be identified. This team may comprise emergency room physicians or cardiologists in small community hospitals, or an intensivist, cardiologist, or cardiac surgeon in larger hospitals with cardiothoracic units. Nursing staff with experience in the care of critically ill patients is necessary. Once assembled, the spoke team should forge a relationship with a hub center for management of acute shock patients. Key aspects of the relationship must include mode and timing of communication and creation of a seamless transfer process.

\section{Mode and Timing of Communication}

The spoke team members should have continual access to the hub surgical team with on-call schedules, contact information, and a clear direction of how to reach the appropriate person, which must be understood by both 
parties. Communication only should occur from a preestablished spoke team member to the hub surgeon and not through intermediaries. The latter approach risks transfer of insufficient or inaccurate information and delays initiation of a definitive plan.

After discussing initial management details, most often, the spoke center will secure the airway and start vasopressor therapy to stabilize the patient sufficiently to allow for safe transfer. Institution of IABP support and high-risk percutaneous intervention will be discussed if available and appropriate. Minimal requirements that must be met before transfer must be fully understood and typically include hemodynamic stability (ie, no ongoing cardiopulmonary resuscitation with reasonable mean blood pressure), absence of ongoing malignant ventricular arrhythmias requiring electrical cardioversion, and absence of obvious contraindications for mechanical support (eg, advanced age, no social support, metastatic cancer).

When available, comorbidities, psychosocial support, patient and family wishes and financial factors should be shared with the hub team so that candidacy for long-term VAD placement and/or transplantation can be ascertained, emphasizing the critical identification of all medical conditions that can impact the care of the patient at the hub institution. In a study of 39 patients transferred post-VAD to a hub center, newly identified medical conditions were discovered in 26 patients [5]. Undisclosed findings (eg, heparininduced thrombocytopenia, recent malignancy, lack of social support) can dramatically change the options available to the patient, and can engender a sense of mistrust between the medical team and the patient's family, as well as threaten the viability of the hub-spoke relationship.

\section{Transfer Process}

The mechanistic aspects of the patient transfer are critical to a successful outcome and must be agreed upon at the outset of the hub-spoke relationship. Issues that need to be defined include identification of an appropriate size ambulance that can comfortably house the patient stretcher, infusion pumps, ventilator, mechanical support console (if present), and personnel. Which institution, whether spoke or hub, will provide this ambulance also should be addressed. The personnel responsible for the transfer must be identified (ie, should they be members of the hub or spoke institution). We believe that at a minimum the transfer team should include a nurse skilled in the management of the ventilator and drug infusions, a perfusionist who can troubleshoot the pump support system and/or IABP (if present), and a physician with experience in critical care. The hub team should provide feedback on the outcome of each transferred patient. Educational sessions led by hub members are critical to strengthen the hub-spoke relationship and to improve the condition of these critically ill patients before transfer to optimize chances of a successful outcome.

\section{Conclusions}

Regardless of etiology, acute refractory shock remains one of the most challenging conditions to treat in contemporary medicine. Left untreated, it inevitably leads to patient demise. Timely institution of mechanical support provides hope for survival in these patients. Efforts to improve the dismal outcomes of acute refractory shock must focus on early referral to regional transplant/VAD centers where multidisciplinary expertise and resources can be employed rapidly.

It cannot be overemphasized that, regardless of device employed, substantial morbidity and mortality always will accompany use of these systems when they are deployed in patients in extremis. The currently available systems share need for systemic anticoagulation, ease of operation, and limited patient mobility. They vary in cost, size, mode of operation, biventricular capability, and percutaneous implantability. To our knowledge, cost effectiveness has not been demonstrated for any of these systems, and comparative effectiveness studies beyond comparison to IABP support have not been carried out yet. Nonetheless, our observations suggest that the unequivocal preference for smaller continuous-flow devices instead of larger implantable pulsatile devices is increasingly applicable to short-term support systems. Should percutaneous systems providing full biventricular support be developed, they likely will emerge as the dominating mode of support for the patient with acute refractory shock.

Disclosures Dr. Daniel Goldstein serves as a consultant to Thoratec Corporation and has received payment as a member of the clinical events committees of Paracor Medical Inc. and Terumo Cardiovascular Systems. S. Neragi-Miandoab: none.

\section{References}

Papers of particular interest, published recently, have been highlighted as:

- Of importance

1. Nieminen MS, Bohm M, Cowie MR, et al.: Executive summary of the guidelines on the diagnosis and treatment of acute heart failure. The Task Force on Acute Heart Failure of the European Society of Cardiology. Eur Heart J 2005;26:384-416.

2. Adams KF, Fonarow GC, Emerman CL, et al.: Characteristics and outcomes of patients hospitalized for heart failure in the United States: rationale, design and preliminary observations from the first 100,000 cases in the Acute Decompensated Heart Failure Registry (ADHERE). Am Heart J 2005;149:209-16. 
3. Helman D, Oz MC: Developing a comprehensive mechanical support program. J Card Surg 2001;16:203-8.

4. Morris RJ, Pochettino A, O'Hara M, et al.: Emergent mechanical support in the community: improvement with early transplant center referral. J Heart Lung Transpl 2005;24:764-8.

5. Gonzalez-Stawinski GV, Chang AS, Navia JL, et al.: Regional referral system for patients with acute mechanical support: experience at the Cleveland Clinic Foundation. ASAIO J 2006;52:445-9.

6. Hunt SA, Abraham WT, Chin MH, et al.: ACC/AHA 2005 Guideline Update for the Diagnosis and Management of Chronic Heart Failure in the Adult: a report of the American College of Cardiology/American Heart Association Task Force on Practice Guidelines. Circulation 2005;112:e154-e235.

7. Schenk S, McCarthy PM, Blackstone EH, et al.: Duration of inotropic support after left ventricular assist device implantation: risk factors and impact on outcome. J Thorac Cardiovasc Surg 2006;131:447-54.

8. Fitzpatrick JR 3rd, Frederick JR, Hsu VM, et al.: Risk score derived from pre-operative data analysis predicts the need for biventricular mechanical circulatory support. J Heart Lung Transpl 2008;27:1286-92.

9. Potapov EV, Stepanenko A, Dandel M, et al.: Tricuspid incompetence and geometry of the right ventricle as predictors of right ventricular function after implantation of a left ventricular assist device. J Heart Lung Transpl 2008;27:1275-81.

10. - Fitzpatrick JR 3rd, Frederick JR, Hiesinger W, et al.: Early planned institution of biventricular mechanical circulatory support results in improved outcomes compared with delayed conversion of a left ventricular assist device to a biventricular assist device. J Thorac Cardiovasc Surg 2009;137:971-7. This is an excellent article involving 266 patients reviewing the benefits of proactive institution of biventricular support versus "watchful waiting" of right ventricular function and delayed insertion of RVAD support.

11. McGee EC, Jr., McCarthy PM, Moazami N: Temporary Mechanical Circulatory Support. In: Cohn L, editor. Cardiac Surgery in the Adult. New York: McGraw-Hill, 2008:507-534.

12. Goldstein DJ, Oz MC: Mechanical support for postcardiotomy cardiogenic shock. Semin Thorac Cardiovasc Surg 2000;12:220-228.

13. Noon GP, Ball JW, Jr., Papaconstantinou HT: Clinical experience with Biomedicus centrifugal ventricular support in 172 patients. Artif Org 1995;19:756-60.

14. John R, Liao K, Lietz K, et al.: Experience with the Levitronix CentriMag circulatory support system as a bridge to decision in patients with refractory acute cardiogenic shock and multisystem organ failure. J Thorac Cardiovasc Surg 2007;134:351-8.

15. Haj-Yahia S, Birks E, Amrani EJ, et al.: Bridging patients after salvage from bridge to decision directly to transplant by means of prolonged support with the CentriMag short-term centrifugal pump. J Thorac Cardiovasc Surg 2009;138:227-30.

16. Loforte A, Potapov E, Krabatsch T, et al.: Levitronix CentriMag to Berlin Heart Excor: a bridge-to-bridge solution in refractory cardiogenic shock ASAIO J 2009;55:465-8.

17. De Robertis F, Rogers P, Amrani M, et al.: Bridge to decision using Levitronix CentriMag short-term ventricular assist device. J Heart Lung Transpl 2008;27:474-8.
18. Kouretas PC, Kaza AK, Burch PT, et al.: Experience with the Levitronix CentriMag in the pediatric population as a bridge to decision and recovery. Artif Org 2009;33:1002-4.

19. Jett GK: ABIOMED BVS 5000: Experience and Potential Advantages. Ann Thorac Surg 1996;61:301-304.

20. http://www.abiomed.com/products/ab5000.cfm. Abiomed, 2007

21. Anderson MB, Plate JM, Krause TJ, et al.: Peripheral arterial cannulation for Abiomed BVS 5000 left ventricular assist device support. J Heart Lung Transpl 2005;24:1445.e1-1445e3.

22. Gregoric ID, Jacob LP, La Francesca, et al.: The TandemHeart as a bridge to a long-term axial flow left ventricular assist device (bridge to bridge). Tex Heart Inst J Texas 2008;35:125-9.

23. Chandra D, Kar B, Idelchik G, et al.: Usefulness of percutaneous left ventricular assist device as a bridge to recovery from myocarditis. Am J Cardiol 2007;99:1755-6.

24. Mehta S, Pae W: Conversion of the TandemHeart percutaneous ventricular assist device to long-term support. Interact Cardiovasc Thorac Surg 2006;4:444-5.

25. - Kar B, Gregoric ID, Basra SS, et al.: The percutaneous ventricular assist device in severe refractory cardiogenic shock. J Am Coll Cardiol 2010 Oct8 [Epub ahead of print]. This article details the most extensive experience with the use of a percutaneous device (117 patients) for the treatment of shock refractory to IABP and vasopressor support. Laudatory effects on hemodynamics, end-organ function, and survival for this moribund population of patients are described.

26. Brinkman WT, Rosenthal JE, Eichorn E, et al.: Role of a percutaneous ventricular assist device in decision making for a cardiac transplant program. Ann Thorac Surg 2009;88:14627.

27. Siegenthaler MP, Brehm K, Strecker $\mathrm{T}$, et al.: The Impella Recover microaxial left ventricular assist device reduces mortality for postcardiotomy failure: a three-center experience. $\mathrm{J}$ Thorac Cardiovasc Surg 2004;127:812-22.

28. Guigis M, Kumar K, Zieroth S, et al.: Interprovincial spoke-tohub transport using the Impella Recover LP 5.0 left ventricular assist device as a bridge to long-term circulatory support. Can J Cardiol 2010;26:320-2.

29. Lam K, Sjauw KD, van der Meulen J, et al.: A combined surgical and percutaneous approach through the axillary artery to introduce the Impella 5.0 for short term circulatory support. Int J Cardiol 2009;134:277-9.

30. Lamarche Y, Cheung A, Ignaszewski A, et al.: Comparative outcomes in cardiogenic shock patients managed with Impella microaxial pump or extracorporeal life support. J Thorac Cardiovasc Surg 2010 Sept 27 [Epub ahead of print].

31. - Seyfarth M, Sibbing D, Bauer I, et al.: A randomized clinical trial to evaluate the safety and efficacy of a percutaneous left ventricular assist device versus intra-aortic balloon pumping for treatment of cardiogenic shock caused by myocardial infarction. J Am Coll Cardiol 2008;52:1584-8. Though a small study, this is the first randomized evaluation of safety and efficacy of a novel microaxial device versus conventional treatment with an IABP. Superior hemodynamics were demonstrated, though no impact in 30-day survival was seen. 